\section{Combinatory Play and Infinite Replay \\ Underdefined Causality in the Neon Genesis Evangelion Anime Series and Games}

Selen Çalık Bedir

The Neon Genesis Evangelion (EVA) anime series has attracted a great deal of attention, first and foremost with its finale. The 26th episode significantly thwarted viewers' expectations, with the protagonist failing to become the legend promised by the opening song of the series and the animation itself struggling to maintain its identity in a jumble of barely moving lines and drawings. Interestingly, with this twist at the end, EVA has also laid bare the presence of a narrative engagement model that does not acknowledge the centrality of endings. Despite the frustration that the series' finale must have triggered in some, the original fate of the characters did not stop the viewers from imagining them in alternative scenarios, just as it did not stop the official creators revisiting and replaying the same scenario.

In the scope of this chapter, it is argued that this particular 'gamelike' narrative engagement (which is built on replays) is reinforced by the EVA video games. The contemporary media environment is marked by an intensity of exchange that takes place not only among media, but also between objects and subjects, forming an extended web of circulation involving producers, consumers and critics alike. This situation requires one to consider a multitude in relation, even when the aim is to focus on the analysis of one specific medium or an individual work. Since 1996, a wide variety of EVA games has been released, ranging from Visual Novels to Dating Sims, Fighting games to Role-playing games, digital Mahjong to card games and so on. Instead of offering a comprehensive historical account, this chapter focuses on a small selection of video games to paint a clear picture
How to cite this book chapter:

Çalık Bedir, S. 202I. Combinatory Play and Infinite Replay: Underdefined Causality in the Neon Genesis Evangelion Anime Series and Games. In: Santiago Iglesias, J. A. and Soler Baena, A. (Eds.). Anime Studies: Media-Specific Approaches to Neon Genesis Evangelion. Pp. 297-326 Stockholm: Stockholm University Press. DOI: https://doi.org /IO.I6993/bbp.j. License: CC-BY 4.0 
of their peculiar interrelation with the narrative of the anime series. In doing so, the author attempts to develop a framework (which takes medium-specificities into account without necessarily prioritizing them) in order to address media relations in franchises such as EVA and to locate the centrality of a specific medium (if there is any), which comes forward as the anime series in EVA's case.

In the scope of this chapter, 'gamelikeness' as a concept is derived from Hiroki Azuma's writings on the consumption of narratives in the postmodern age, and the medium-specificity of video games is explored in reference to Game Studies. In order to compare and contrast gaming experience with the experience of engaging with narratives, the author borrows Brian Upton's concept of 'play,' defined as "free movement within a system of constraints" (20I 5: I 5). Upton applies this concept to narrative and ludic engagement alike, noting that narrative play takes place in the mind as an interpretive and anticipatory process. Revising Upton's theory in the light of Azuma's, this chapter suggests considering narrative replay as 'combinatory play,' which is defined here as "free combination of narrative elements allowed by a suspension of causal constraints." Finally, as the video games' relationship with the anime series is discussed in connection with the weakening of causality in contemporary narratives and with serialization, this particular study can be categorized under Media Studies with a narratological bent.

Azuma brought together narrative media with games to discuss the significant change in audience engagement with popular narratives in Japan, a trend which seemed to have emerged with EVA itself. Employing the concept of 'play' to lay a solid ground for comparison and including the medium-specificity of games in this discussion yield even more insight into audience engagement in general. Taking these steps centers the discussion around dominant patterns of causality management. These patterns are dominant, but not absolute, as they are constantly contested and prone to change in relation to the 
constant interaction between media. The following discussion posits that, in this case, within the EVA franchise, the anime series is not only the start, but also the reference point in setting the model for causality management, according to which certain medium-specific features of the EVA games seem to be set aside.

\section{Severing Characters from their Contexts}

In Otaku: Japan's Database Animals, Hiroki Azuma attracts attention to a rupture in audience engagement with narratives, which he demonstrates by comparing the wants and needs of Gundam ${ }^{1}$ and EVA fans.

[N]umerous fans of Gundam desired the completion and close examination of a singular Gundam world. That is to say, in their case they preserved the current passion for a fictitious grand narrative. However, even during the peak of the craze, the fans of Evangelion who appeared in the mid-I990s-especially those of the younger generation (the third generation of otaku) — did not really have a concern for the entire world of Evangelion. Instead, they focused exclusively on the settings and character designs as objects for excessive interpretation or 'reading up' (exemplified in derivative works), and for chara-moe. (2009: 37)

In Azuma's account, EVA fans stood apart from earlier diehard consumers with their disinterest toward the totality of the narrative they were consuming, or toward how the characters, their world and whatever should happen in that world (the main events of the plot) were particularly interwoven. The real source of attraction for them was any sort of EVA-related data that they could enjoy separately or in relation to other characters in different diegetic contexts.

Azuma relates this consumption tendency to Jean-François Lyotard's theories on the postmodern condition, especially to the disappearance of grand narratives. To put it briefly, along with postmodernity (which came to full bloom, according to Azuma, with the collapse of communism in I989), widely accepted social ideals
I. One of the biggest media franchises from Japan, which started off with the mecha (giant robot) anime series Mobile Suit Gundam (created by Yoshiyuki Tomino, released between I979 and I980). 
that organized human life turned untenable. As a result, storyworlds seemed to be less and less guided by organizing principles as well, gradually leading to a disintegration of the once very closely knitted characters, setting and plot. In tandem with the advancing information storage and sharing technologies, especially with the widespread use of the Internet, both producers and consumers have grown familiar with massive sets of elements that compose narratives and gain meaning as they are combined. The perception of each work as a particular combination of such compatible 'database elements' has granted narrative consumption a gamelike quality as well, in the sense that it has enabled everyone to replay a finalized plot if they choose to, by taking the characters out of their diegetic contexts and building them new ones with an alternative set of pieces.

The possibility of taking characters out of their diegetic contexts may seem like a stark contrast between the mindsets producing contemporary popular narratives and works of realist literature where characters' fates are sealed by authors. However, it can be claimed that the birth of the circumstances loosening up this character-context connection (resulting in character mobility) goes back to the I9th century and manifests in a certain sense in realist narratives first. In The Future of the Image, Jacques Rancière points out that the proliferation of information, which reveals itself with the widening use of printing technologies and culture (2007: 16), finds its reflection in realist novels as a confusion of causal connections (2007: I2I). These novels revolve around anything and everything that can happen in real life, and picture the mundane details of daily life alongside the phenomena that cripple human mind and agency in their gravity (i.e., traumatic events, such as war). In this manner, first of all, realism paves the way for combining narrative elements which are unlikely to come together. However, at the same time, it becomes particularly challenging for the reader to untangle the connections between them, hence to pinpoint the exact causes and to anticipate the exact outcomes of actions in the 
depicted storyworlds. Be that as it may, the grand narratives still functional back in the isth century set some limitations to hold the characters in check in realist literature. In Oliver Twist, for example, despite the fact that Charles Dickens gives the protagonist's name to his novel, the socio-economical status of Oliver becomes the most impactful factor defining how his fate unravels, as well as the most significant limitation which can be overcome only through meticulously interrelated chance encounters. This particular, uniquely woven contextual fabric makes Oliver who he is, and it is through Oliver that one can understand the figurative mold which gives him this shape.

At first glance, the EVA anime series seems to preserve the charactercontext connection when it puts an unexpected end to the journey of the characters as the context collapses literally in an apocalyptic scenario. The chaos that reigns in their world correlates with the characters' psychological as well as physical condition, making it impossible for them to take a stable position or form. However, perhaps as it is signaled by the fact that apocalyptic incidents are not unprecedented in the EVA universe (the story takes place I 5 years after a cataclysm after all), the ending does not prove to be a point of termination. While the collapse of the diegetic context results in the disintegration of characters, characters' clear-cut depictions (which allow them to be recognized via any of their most dominant characteristics in any context) and the centrality of their emotions in the narrative (which occupies the attention that would otherwise fall on the other elements surrounding the character) prove enough to summon them back to life. As a result, it can be said that characters overshadow the context and emerge as the orbital axis of causality, making it possible to break down and reconstruct the chains of action and outcome in whichever way necessary to present attractive characters in an affect-inducing manner. Used to the wildest combinations of narrative elements already, both creators and audience engage with EVA in a gamelike manner, reimagining characters in different contexts regardless of 
whether or not it was the original intention of the director Hideaki Anno in the first place.

\section{Character-Driven EVA Games}

How about the EVA games? How are they aligned with the anime series that displays the above described gamelikeness? What kind of audience engagement do they offer? And how does this reflect back on the audience engagement with the anime?

As a wide variety of EVA games has appeared since $1996,{ }^{2}$ it is in fact quite difficult to make sweeping statements while addressing the above questions. Luckily, an earlier study on the relation of video games and another popular anime-manga series, NARUTO, provides an excellent starting point. Studying four NARUTO video games in detail, Martin Roth demonstrates the inapplicability of Azuma's theory to video games, particularly with regard to the formation of characters. Roth states that game characters should be considered "beyond their appearance and names, little more than empty shells that can be filled with content or functionality as demanded" (20I3: 25 I). In Azuma's understanding, the characters of gamelike narratives feed on a huge database of distinguishable features. The video games discussed by Roth maintain the looks of the NARUTO characters, which produces a direct visual connection between the games, the manga and the anime series. However, it might be said that the characters in the discussed games are pretty much hollowed-out versions of the original characters. Roth explains that particularly in fighting games, for programming reasons and for balancing up the experience of playing with one character instead of another, the behaviors assigned to characters largely overlap in a fashion that erodes characters' particularities beyond their looks. Moreover, gamers may be allowed

2. Over 30 titles according to the Computer Entertainment Rating Organization (CERO) website. to challenge friendly characters on the battlefield or to form unlikely alliances with sworn enemies, despite how unimaginable it would be to see such actions performed in the original plots. 
With a selection of titles featuring battle modes at the very least, Roth also demonstrates that video games do not have to tell stories. This fact, though apparent, is necessary to underline especially in cases where the experience of playing games is compared to audience engagement with narratives. The term 'narrative' can be taken as the semiotic representation of a world, populated by characters and displaying change over time, as well as a sense of causality. ${ }^{3}$ In this sense, it is possible to talk about narrativity in video games as well, despite the once popular divide between this particular medium and narrative media. At the beginning of the 2000 s, certain scholars (self-labeled as the ludologists) reacted to the tendency they spotted in narratological analyses to reduce video games to just another storytelling medium, and advocated centralizing the distinguishing features of the medium instead. The ensuing ludology-narratology debate settled over time with the emergence of more nuanced approaches underlining the potential of the medium to tell stories, while recognizing the primacy of its interactivity. ${ }^{4}$

NARUTO games intersect with the anime and manga on several occasions as they allow players to control and interact with a long list of characters from the entirety of the series. But as Roth demonstrates, some of these games (especially of the fighting genre) and some modes offered within separate games mainly aim to engage players in a cyclical interactive experience: to explore the range of possible actions enabled by the game system, pick the most advantageous ones to perform and carry on doing so based on the output produced by the system. The fights themselves may not reveal extra data about the storyworld or make a significant contribution to story progress; rather than that, they prioritize the practice of honing gaming skills crucial to the in-game progress. The pleasure taken from such games has a direct connection with the quick display of control skills, and playing NARUTO games makes a distinct contribution to the engagement with the whole media franchise thanks to this particular experience it offers. Beyond the looks of the characters, the game establishes its
3. For a detailed definition, see Ryan (2003: para. I).

4. For a detailed account, see Simons (2007). 
relationship with the original story via cutscenes (video clips) interspersed between fights. However, these cutscenes are not necessarily presented in temporal or causal connections with the fights either. After dealing the last punch in the ring, a character can appear in a completely different setting engaged in a completely different action. These examples reveal clearly that narrative coherence is not among the primary objectives for such games.

These observations by Roth can also be applied to Neon Genesis: Battle Orchestra. Battle Orchestra is a fighting game that comes with a rumble mode enabling up to four different Evas (the mecha controlled by the main characters of the series) or Angels to attack each other without necessarily abiding by the 'facts' set straight by the series. But among the rest of the EVA games there is a significant number of narrative-oriented and character-driven titles. The prime examples of these would be Visual Novels (such as Girlfriend of Steel I and 2) and Raising simulations (Ayanami with Asuka Raising Project, Shinji Ikari Raising Project, etc.) that centralize the engagement with the characters from the series and excite EVA fans, especially with the opportunity to form a direct connection with exactly who these characters appear to be in the series. Azuma defines visual novels as "multistory, multiending novels ... that can be 'read' on the computer screen with images and sounds" (2009: 75). Raising simulations, on the other hand, put players in a more active position which requires observing a high number of interrelated variables and overseeing characters' growth by carefully balancing their use of limited resources (i.e., time that can be spent on certain actions). To generalize, the common goal of these more character-centered EVA games becomes traversing the game and observing the characters in different contexts as a result of players' well or poorly made decisions. While control skills are largely swapped with instances of clicking, the physical performance of the players is replaced with their capacity of perseverance for the love of discovery. However, interestingly, despite the fact that this is precisely the structure that inspires Azuma to develop his theory of narrative 
consumption revolving around the primacy of the character, we can find a sort of 'characterlessness' in these games as well.

For example, an online event guide describes one of the possible routes for character development in Ayanami with Asuka Raising Project in the following fashion: ${ }^{5}$

\section{Conditions:}

Sensitivity is above 200

Fatigue is higher than morality

Comments:

Have you ever wondered what Rei [Ayanami] would be like with Asuka's personality? Well, get this state and you can find out! You'll know when you've gotten this when Rei poses, her head held high and a confident smirk on her face. She'll occasionally disobey poor San'i and go off and do her own thing (despite the schedule) but she's still kind at heart. I believe there's an event where she runs away from home and comes back when San'i is angsting while it's raining heavily, but I'm not too sure how to trigger it. (GrunGast82 2004)

In order to describe the consequences of reaching the above-listed conditions, the author of the guide suggests that we imagine how Rei would look if she had the personality of Asuka. In other words, the author invites us to imagine a familiar character acting completely out of character, saying things another character would say with the expressions commonly seen on that other character's face. Although this may sound like a total erasure of character, it may also be considered the proof of how a character can be reduced to her most defining parts. In this case, Rei's appearance (minus her usual facial expressions) is chosen from the sum of her character traits established through the series. Moreover, the same event guide also reveals that choosing to engage with Asuka in the same game basically presents the same outcomes if the gamer is to follow the same instructions. ${ }^{6}$ This being the case, it is only the visuals that should convince us that Asuka (instead of Rei) is the one we are engaging with.
5. The following quote employs informal use of English. Grammar mistakes are left unedited on purpose.

6. Final note of the guide's author reads: "All the above also apply to Asuka, except Asuka's normal state (low social skill) can be described as 'Grumpy,' while her normal state (high social skill) is the Asuka we all know and love." 
It is safe to assume that playing the same game with a second character would not be that challenging or enjoyable for a person who is interested in mastering simulations in general. Mastering this particular simulation means reaching favorable states by closely observing the interrelation between variables such as sensitivity and fatigue levels and making decisions accordingly. The second run of the same game, on the other hand, should be rewarding rather for those who aim to see precisely such variations of Asuka (available combinations). The very first sentence of the excerpt above finds its target audience right away: this game is right for those who have wondered what Rei would be like with Asuka's personality.

\section{Causal Connections in Story Generating Games: The Case of Neon Genesis Evangelion 2}

Among the EVA games, another title that stands out is Neon Genesis Evangelion 2. NGE2 is a very complex dating simulation (which requires a skillful management of resources to win the non-playable characters' affection) interspersed with (real-time) combat. During combat, the controls grow more challenging than simple clicking, and players need to pay attention to timing while developing their strategy. Consequently, the branching of the story is not solely decided by the choices of the gamers, but also by the results of the battles and the additional points collected throughout the game. In comparison to the Raising simulations discussed above, there is a higher number of conditions to meet in NGE2. But this complexity comes with its own advantages.

NGE2 bases its reward system on the discovery of alternative scenarios circling around familiar characters, spiced up with the opportunity of forming romantic relationships with them. First of all, the PlayStation Portable (PSP) version of the game released by Bandai in 2006, Neon Genesis Evangelion 2: Tsukurareshi Sekai-Another 
Cases, contains extra scenarios, as well as revisions of those presented two years earlier in the PlayStation 2 version. Second, both versions of the game reveal some information allegedly sanctioned by Anno himself; the canonicity of the so-called 'Classified Information Files' is debated, but intriguing in any case. Third, according to a statement of NGE2's system project manager, the team wanted to incorporate Anno's view of EVA into the game, reflected by the fact that the system itself was named Anno AI.7 Lastly, the same press release reports Anno himself saying that while he is not planning to make a sequel to $E V A$, he would like to see the audience produce their own EVA story via this game (Dengeki Online, 2003). This statement alone shows how NGE2 is supposed to connect us directly with the series by functioning as a call to write one's own EVA story with the data from the series, but also echoes the narrative consumption style that leads the audience to imagine what else could have been.

NGE2 and the EVA series are purposefully connected in the manner described above. However, it doesn't mean that these differing media take the audience through the same process. The diversity among games (from games with a clear goal to those with no winning states, games that require the use of certain materials as opposed to those that don't, games of chance, make-believe, sports, etc.) makes it quite challenging to come up with a simple and inclusive definition. By taking a comparative look at the available definitions and listing the most common traits, Katie Salen and Eric Zimmerman propose the understanding of game as "a system in which players engage in artificial conflict, defined by rules, that results in a quantifiable outcome" (2004: 80). While this is a good definition, as games can also tell stories, counterposing them to narrative media is one other step to delineate the limits of the medium further. One of the big names in Game Studies to discuss medium-specificities, Espen J. Aarseth, starts his seminal work Cybertext with an attempt to exemplify the main feature setting narrative engagement apart from play.
7. It is even recommended to imagine the thoughts of the director when playing the game the second time (Dengeki Online, 30 October 2003). 
A reader, however strongly engaged in the unfolding of a narrative, is powerless. Like a spectator at a soccer game, he may speculate, conjecture, extrapolate, even shout abuse, but he is not a player ... He cannot have the player's pleasure of influence: "Let's see what happens when I do this." The reader's pleasure is the pleasure of the voyeur. Safe, but impotent. (1997: 4; emphasis in the original)

At first glance, the focus in Aarseth's comparison seems to fall on the passivity of the readers who show only a trivial physical effort to witness story development, but cannot influence the course of action. However, there is one more point of equal importance. 'The player's pleasure of influence' seems to depend on the system's ability to produce the outcome of the action that players initiate. Following the footsteps of Aarseth, Gonzalo Frasca presents traditional narratives as the exact counterpart of simulations and underlines the fact that games enable objects to react to the input provided by the gamer by modeling their behaviors:

Traditional media are representational, not simulational. They excel at producing both descriptions of traits and sequences of events (narrative). A photograph of a plane will tell us information about its shape and color, but it will not fly or crash when manipulated. A flight simulator or a simple toy plane are not only signs, but machines that generate signs according to rules that model some of the behaviors of a real plane. (2003: 223-224)

As these quotes attest, first of all, both Aarseth and Frasca seem to consider the game players' freedom to explore in-game possibilities and the control they have over (characters') actions as the prominent features of ludic engagement. However, as discussed earlier, the consumption of gamelike narratives gives a considerably more active role to the audience. The consumption model presented by Azuma goes beyond an individual's comprehension of 'descriptions of traits and sequences of events'; it allows and encourages the audience to go through several reiterations of the same story and to modify it if desired. Therefore, in the comparison between games and gamelike narratives, 
the following fact underlined in both of the above quotes plays a more decisive role: games can simulate the outcome of an action (falling within the range of actions enabled by the game system) that the gamer prefers to perform. The outcomes of the gamer's 'non-trivial efforts' (which may go beyond decision making, and involve skills of problem solving, coordination, etc. depending on the game) are calculated according to a certain set of rules that regulate possibilities in the gameworld (modeling). These outcomes may or may not display narrative value.

At this point, let us turn to another unofficial online guide, this time for the extended NGE2 for PSP, to have a better understanding of how the NGE2 games work. ${ }^{8}$

- SCENARIO O2: Shinji Ikari -

TITLE: "I like this world, though"

MAIN TARGET: Get along with every other character and beat the EVA Series.

CHARACTER SKILLS: Work (Io), Knowledge (Io), Self Defense (O2) and Synchro (IO)

CHARACTER STATS: A.T.: 42 / IMP: 90

SLEEPING TIME: From oI a.m. to o7 a.m.

In this Scenario, Shinji must try to get along with every other character (except PenPen), so he can feel more comfortable with his life in Neo Tokyo-3. Shinji must raise his relationship values with everyone at +700 , and everyone must feel for him the same high relationship values. This is quite tough to achieve, especially without the help of any special item like "Unbreakable Heart."

Once you reach +700 values with someone who also feels +700 values for Shinji, a scene will activate in which Shinji and the character share an intimate (and usually fun) event that develops their friendship. These events will only appear when you enter at the required place for each character:

Misato's apartment (before 4 p.m.): Misato, Asuka and Kaji. School (studying time): Hikari, Kensuke and Gendou.
8. The following quote employs informal use of English. Grammar mistakes are left unedited on purpose. 
Convenience Store: Hyuga (7-I I p.m.) and Touji (3-9 p.m.). NERV: Fuyutsuki, Maya, Aoba, Kaworu, Rei and Ritsuko.

[Jumping to instructions on battle]

The order of the battles is random (except the first against Sachiel), and they can get quite tough depending on your ability, stats, and the help that you get from other Pilots. I recommend you to do a lot of training with other Pilots. Not only it will raise your Synchro, but also will enhance your relationship.

You will know that the EVA Series are about to attack when the DefCon has all turned red but still no Angel attacks. Once you enter NERV, you will watch many scenes. Misato (or your favourite NERV Personnel person) will come to rescue you, and then your Self Defense will be important to survive. (If you happen to die here, reload your game: it's a random event, actually, although the higher Self Defense, the higher probabilities that both you and the Misato will survive.) After this, the battle against the EVA Series will begin. Defeat them to watch the ending.

Note that if any character dies for any reason during this Scenario, you will not be able to get the best ending. (Pler 2008)

This lengthy excerpt proves very helpful for demonstrating how the game system generates a multiplicity of EVA stories based on gamers' non-trivial effort, as well as elucidating the causal connections that the game relies upon. First of all, both NGE2s make it possible to test out the consequences of a wide variety of actions. In this particular scenario found in the NGE2 for PSP, as Shinji needs to raise his relationship values, the player may decide to have him engage in a conversation, or smile at or even kiss other characters at a given time. This freedom of action is way more emphasized if we consider that the scenario above is only one of the 18 scenarios found in the game.

However, as in any other game, only certain actions are possible in this version of NGE2 and only some of these actions are desirable, granted that the aim is opening alternative routes for plot development 
and completing the game. In the given scenario, while it is up to the player to decide what Shinji will do, the character's actions will have to fall within the range set by the game system and should earn him over 700 relationship points for successful completion. These points can be collected by performing one's own selection among a variety of actions which are assigned numerical values, hence are swappable in terms of their long-term, cumulative consequences. But the guidebook also reveals that some actions have direct consequences on whether and how the game will progress. For instance, although it is possible to reach an ending despite casualties, in order to get the good ending one needs to keep every character alive in this particular scenario. Then, if the goal is first to progress and then to discover all the routes for plot development and endings, staying within certain parameters will be the only way to reach that goal. In other words, the general outlines of the narrative and the consequences of narrative significance (breaking points in the fates of characters and their interrelations) are largely predefined.

That being said, randomness does not stay entirely out of the picture either. In the given scenario, in order to reach the listed consequences, one should meticulously follow the guidebook and collect points as advised. Nevertheless, whether Shinji will live to see the final battle depends on a randomized event. In fact, as Greg Costikyan states, randomness plays a bunch of significant roles in games: "it adds drama, it breaks symmetry, it provides simulation value, and it can be used to foster strategy through statistical analysis" (2013: 86). However, as Costikyan immediately adds, incorporating the random is also pretty risky, for "in excess, it imbalances games, it can foster a sense that success is a consequence of luck rather than excellent play, and it can produce frustration when a streak of bad luck affects a player" (ibid.). While adding variation to gameplay (thus individualizing it), as well as reinforcing the element of surprise, randomization reduces the non-trivial effort of the player to an act of chance. 
Therefore, game designers usually aim to strike a good balance in randomization, and harness the sources of uncertainty.

With randomness involved in its I 8 scenarios, NGE2 for PSP presents a wide variety of possible actions and invites gamers to explore alternative routes for plot development again and again for long hours. However, while relying further on randomization can increase replayability even more, both NGE2s opt for keeping it at a certain level. In addition to the warnings by Costikyan, in the case that randomization is maximized in games such as the NGE2s, it is inevitable that asymmetries will arise between the stories to emerge. Due to chance factor, some stories might end simply too early, before any significant change is observed in the state of characters or suddenly after a long and unrepeatable gaming session. Instead, in the NGE2s, causal connections are built in advance to ensure that these sessions can produce meaningful EVA stories with equal dramatic impact to that of the series. Seen from a different angle, the guidebook reveals not only the possibilities enabled by the NGE2 systems, but also the certainties it rests upon. In the given scenario, most obviously, if the players manage to keep Shinji alive with over 700 points, it is certain that the game system will reward them with the promised ending. Then, it is only through this emphasis on causality and by assuring the birth of certain consequences that the NGE2s emerge as storytelling games.

\section{Narrative Play vs. Narrative Replay, or Anticipatory vs. Combinatory Play}

The fact that gamers play an active role as they engage with an interactive medium is mentioned quite often as a specific aspect in research on games. However, it would be misleading to think of narrative engagement as an entirely passive activity. In fact, Brian Upton suggests that engagement with both narratives and games should be considered an experience of play, which he defines as "free movement within a system of constraints" (2015: I 5). From this point of view, 
the activity of interpreting what is happening in a story and anticipating what will happen next becomes the counterpart of gamers' activity that is both limited and enabled by rules. Play in general requires players to map out the potential field of action, but while gamers face the consequences of the actions they choose, readers or cinema goers have their guesses tested out as the story unfolds. In this sense, narrative play becomes an active mental exercise. However, as discussed in the very beginning, gamelike narratives encourage if not necessitate the audience to give a more concrete form to their mental experience by producing alternative stories in the form of derivative works.

In realist literature, although trivial things and events occupy the stage along with grave occurrences, the causal axis for plot development stays very clear. The consequences of actions are supposed to be defined to a great extent by the rigid grand narratives of the I 9 th century, while unlikely developments are meticulously woven into the story as wild twists of fate. With the waning of grand narratives, the possibility of turning readers' mental activity into an autonomous re-creation process has gradually gained visibility. The liberation and materialization of audience experience emerged as an option, thanks to, first of all, the media environment. The increasing accessibility and consumption of narratives enabled the audience to identify the most common elements and patterns in different works. As a result, it became possible to think of new narratives as variations of the already present ones. With every 'new' version of a familiar story, with every new connection between actions and their consequences, it becomes clearer that everything, no matter how unexpected, is potentially explainable. This being the case, everything becomes possible in any given storyworld. Some narratives embrace this possibility and display a blatant lack of causal organization. As the audience's understanding of the field of possible actions grows vague, even when the ending of a story is revealed at its very beginning, the route that will lead to that end remains a mystery. The use of familiar narrative elements is crucial for the process of anticipation, but not entirely enlightening either. 
Even though the use of standardized characters, settings and events seems to narrow down the number of the narratives that can be produced, what they can form together is practically endless as there are no guiding principles determining how they should be combined. In worlds where not only wild coincidences but even contradictions are justifiable (as branching timelines and alternate realities), chaos evolves into infinite adventures, provided that characters are distinguishable enough in any context. Then, the discovery of each particular combination of characters, settings and events becomes an object of audience interest, whereas the connections between them seem temporary from the start and re-built whenever necessary. Instead of asking 'what should happen in this world?' (according to rules that regulate causality) and meticulously meshing unlikely coincidences with the fabric of narrative, it is possible to start from a desirable consequence and loosely pave the way up until that point, skipping steps if desired, knowing that anything can be forced into the gaps left in the process.

Equipped with such a view on narratives, the audience might be interested in building their own set of connections at will as well. If a character is attractive enough, the audience can build an alternative scenario with that character, embarking on new adventures that are not present in the original work or even clearly conflicting with what is depicted officially. This vicious cycle guarantees infinite twists to an audience satiated with stories and provides the space as well as the incentive for the creation of new, surprising stories. Then, the weakening of causal order and its deliberate undermining in gamelike narratives enable the audience to simulate alternative routes for plot development themselves. In this way, they also initiate a form of serialization that differs from the traditional type which takes the shape of prequels and sequels. They encourage everyone to replay the same story and produce alternative versions as derivative works, through abandoning the attempt to establish and abide by causal connections, combining whatever they want to keep from the original work with other narratives of their picking. This form of narrative replay can 
be named 'combinatory play,' that is, free combination of narrative elements allowed by a suspension of causal constraints (as opposed to Upton's interpretive or anticipatory play, which takes place entirely in the mind of the audience).

\section{Combinatory Play across the EVA Games}

Pursuing alternative directions for plot development in narrative play becomes an end in itself and brings the experience of engaging with narratives one step closer to the experience of playing games. In the absence of guiding principles concerning the consequences of actions, along with the unraveling of connections between characters and contexts, everything seems equally possible in any storyworld and complete rewrites become valid forms of serialization. Gamelikeness ensues in that these narratives allow the audience to take control of characters' actions in the alternative stories of their own making. However, this type of engagement differs from playing games in that the audience can also control the outcomes of actions. This results in the possibility of producing alternative stories starting from their end. In other words, rather than asking what would happen in a particular world, one can fill in the background of any desirable situation. Instead of delineating characters' capacity of action under certain circumstances, the production of gamelike narratives depends on a level of recognizability in character design that is also transferable to the parts that constitute a character. This allows characters to preserve the core of their identity in any context and do whatever they like.

The previously cited online guide of NGE2 for PSP demonstrates how both versions of the game differ from gamelike narratives precisely in these respects. The NGE2s allow players to observe what would be possible in the EVA world, based on the way in which this world is modeled (i.e., organized by a relatively simplified set of rules) in each case. Moreover, as they are intended to be story generators, the NGE2s fix causal relations to a great extent and minimize 
randomness to ensure that noteworthy events can be meaningfully aligned in every complete play. Although the wide variety of systemenabled actions makes it highly unlikely for different people to play the games in the exact same way, performing certain actions and reaching certain states (as a result of carrying out sets of actions that may vary in themselves) will produce the same consequences for all. The NGE2s provide players with alternative stories of emotional impact, precisely by setting the range of what in-game objects can do, and by predetermining causal relations to an extent through assigning direct consequences to some actions and states. But surprisingly, taken as a series, the EVA games offer an experience bearing an unexpected similarity to that offered by gamelike narratives.

Considering the fact that there is a wide variety of EVA games, it is difficult to approach them as a set. The EVA games differ in genre and in the ways they connect with the anime series. Detective Evangelion, for example, is an Adventure game which surprisingly turns Shinji Ikari into an investigator of some murders that do not exist in the anime series. However, despite the variations, a common trait that cuts across the EVA games is worthy of notice: almost each of them borrows a certain set of elements from the anime. Not only do we see familiar characters appearing in familiar settings (sometimes in the company of newly introduced faces), we also see these characters once again grappling with the same traumatic events from the series. In the Raising simulations (such as the Ayanami with Asuka Raising Project), the attacks of the Angels are still a part of the characters' otherwise ordinary lives. Those who play these Raising sims are responsible for characters' clothing, as well as their battle training. In Detective Evangelion as well, despite the fact that the game scenario blatantly digresses from the EVA anime series, we see that the protagonist is still fighting Angels as he tries to solve murders.

As discussed previously, games don't have to tell stories or maintain consistency when they tell stories, just as in the NARUTO games 
discussed by Roth in detail. But the EVA games obsessively relate to the same story, by featuring the same characters, settings and main events from the anime series. Even Evangelion New Theatrical Edition: 3nd Impact, which is a Rhythm game that requires players to trace the beat of a song or sound clip, goes directly back to the series with each correct move and reveals familiar images or video clips. Moreover, at certain levels, the game switches to monologues from the series instead of music, which transforms the experience into an information hunt ('Which parts am I going to see? Will there be anything new?' being the concerns) instead of an ordinary music game simply employing the EVA soundtrack. The games, therefore, seem to engineer an audience engagement that is most enjoyable in reference to the anime series.

This referential loop is actually established by the anime itself. At its end, the anime series reveals flickers of alternative directions for plot development, which spiral into rewrites in the shape of animated movies and other works. Despite the high number of overall EVA titles, rather than the addition of new story arcs, the official franchise expands via alternatives of the same story. This cyclical serialization produces a very small pool of EVA-related elements to rely upon in the creation of new works, and the surprise value in these works does not come from what will be combined as much as it comes from how the same elements will be combined. The EVA games take part in the same practice, as specific narrative elements are interwoven in each game especially around the fight with Angels. This step shapes the engagement with the games to a noteworthy extent.

If "traditional narrative media lack the 'feature' of allowing modifications to the stories," as Frasca states (2003: 227), gamelike narratives trigger the materialization of alternatives in the next work to follow. Pursuing alternative stories in games such as the NGE2s, on the other hand, is a requirement rather than a mere possibility according to Frasca. However, interestingly, although it is possible 
within the same game to picture different paths for plot development, these games tend to go back to the same iconic EVA battles one after another to retrace or reimagine the relationships between a selection of the same characters in separate works.

While it is always an option to use the same mechanics or modeling in serializing games, which is not only cost-effective, but also preferable for establishing a particular style, these games keep changing both elements and explode the possibilities of interaction with the same series across time and different genres. The EVA games do not rely on downloadable content (DLC) to prolong consumer engagement either. Through DLCs, new objects or environments can be introduced in installments without changing the mechanics or the rule system of a game. However, instead of capitalizing on this strategy, the EVA games seem to connect over a specific set of narrative elements as the games keep increasing in number. (Bandai-Namco's Tales of Zestria JRPG series has an Evangelion DLC pack that comes with a costume set though.)

Even when the gameplay centralizes gamers' non-trivial efforts or the aspect of randomization, connecting with the anime series by featuring the same characters and events creates the expectancy as to what the gamer will do in order to see another version of the same story. To the extent that this concern gets pronounced, the experience with the whole franchise appears narrative-oriented and emerges as a rewriting process. Moreover, the endless reiteration puts storygenerating games such as the NGE2s in a specifically paradoxical position. Although they enable hours of replay in themselves, this quality seems limited in comparison to the inexhaustible potential of rewriting the same story forever.

The EVA games, in sequence, build an obsessive connection with the anime series by featuring its main characters and events over and over. In this respect, the EVA franchise treats games like narratives, as if they needed serialization to introduce alternative ways for plot development. EVA games like the NGE2s flaunt the medium-specific capacity to simulate the outcomes of possible actions, but this capacity 
gets absorbed by serialization: games that function as story generators seem to rewrite the same story one after the other. The causal infrastructure (that these games build in order to enable the process of creating alternatives) seems like an alternative to infinite others in the first place, and each game seems to herald the coming of the next one already. In this light, setting medium-specificity aside, instigating combinatory play becomes the common point across the video games and the anime series. Regardless of their genres, these games instigate an active discovery of a new set of connections between the same characters and events once again.

\section{Final Remarks and Future Directions}

In literary realism, although chance encounters are not uncommon and in fact quite influential on how actions and consequently characters' fates will unravel, the diegetic context (encompassing physical surroundings as well as socio-economical circumstances) is given an undeniable and unequalled weight in shaping causal connections. To put it differently, while chains of actions and consequences are mostly organized as the context dictates, unlikely yet often observed occurrences are meticulously justified by chance. The resulting configuration (of what happens when and where) draws a unique fate for the characters, pinning them down at a certain position and making them who they are.

Gamelike narratives, on the other hand, start from the premise that everything is justifiable on the condition that characters are welldefined. This type of narrative severs the character-context connection and annuls their interdependence. As characters are attributed recognizable traits from the start, there is no need for them to be defined by a particular context, and conversely, they don't need to function as an anchoring pin for a contextual fabric that could otherwise unravel.

Gamelike narratives leave causal connections underdefined on purpose, in order to allow characters to roam outside their original 
contexts, outside any zones of restriction. Such narratives encourage the audience to engage in a specific type of 'combinatory play,' which can be defined as the free combination of narrative elements allowed by a suspension of causal constraints. As long as there are well-defined characters in a narrative, one can always select a set of favorites and imagine them in new diegetic contexts. Moreover, it becomes possible to completely rewrite these characters' actions, for their scope is not predefined by causal necessity. Gamelike narratives, then, not only unlock the potential of endless serialization, but also (more remarkably) enable the creation of endless alternatives, worlds of infinite possibilities where the exact same action can produce different consequences. EVA is a great example of the latter tendency.

Gamelike narratives seem to get closer to games by allowing for a quest for alternatives via downplaying causality. However, in the case of video games, the range of possible actions is necessarily predetermined to differing extents, so that the system can calculate and present the consequences of a variety of in-game actions and encounters. Standing out among the EVA games as the ultimate story generators, the NGE2s rely on specifically pronounced causal connections (which is not a requirement for all types of games).

$N G E 2$ for PSP offers quite a variety of in-game actions and a high replay value, given that it consists of $\mathrm{I} 8$ different scenarios with multiple endings. However, performing actions randomly is not enough to successfully traverse all the courses for plot development and reach the desired endings. In both versions of NGE2, at certain times, characters must be led to do certain things in a certain sequence. Using Jesper Juul's terminology, the NGE2s come with a great deal of such predetermined 'progression structures,' so that the alternative stories to emerge from play sessions are all rewarding, striking narrative experiences. (Juul sets 'games of emergence' apart from 'games of progression' on the grounds that the former introduce a bunch of rules which loosely define the range of what is possible in a game, while 
the latter require players to follow specific to-do-lists to successfully cover a game. Nonetheless, progression structures can be observed in games of emergence as well, especially in the shape of specific quests or objectives.) At other times, even when the gamer is entirely free to make a pick within a field of possible actions, these actions must still yield enough points to stay within a certain range for progress. Provided that at least one of their aims is to present stories on a par with the anime, the creators of the EVA games need to make sure that there will be significant events with sufficient dramatic impact on the way in which the stories progress.

Of course, not every game needs to centralize the role of narrative in the particular experience it offers. Despite the fact that there is a narrative potential in video games, it is not the goal of each to construct a narrative-driven experience. Through a cross-media analysis of NARUTO, Roth settles down to prove "how software-based videogame adaptations go beyond textual media in that they can facilitate a wide range of ways to play with and without narrative elements by combining franchise elements in ways unique to the medium" (2013: 244). NARUTO games feature familiar faces from the manga and the anime, along with a selection of settings and events from the series. However, Roth underlines that in a game, narrative coherence may be only of secondary importance or not sought after at all. The action and fighting games examined by Roth do not necessarily make significant contributions to the anime or manga storylines. In fact, they even discard some of the unique features of the characters, for their priority falls on creating a balanced environment of challenge, where gamers test their control skills. Nevertheless, be it limited to visual similarities or a strong adherence to plot, any connection with the series presents an extra source of fun for those who are familiar with the original narrative.

Similarly, the EVA games differ in genre and offer players novelties in gameplay as well as in the ways they connect with the anime 
series. Some of these games present the opportunity to play out the exact same scenario as the anime series. Some allow players to carry out new actions with the original set of characters. A final group of games introduce entirely new characters to mingle with the rest. Nevertheless, all of these games present clear connections with the anime series, specifically by featuring a number of the original set of characters along with certain recurring events. Even games like Detective Evangelion, which introduce striking divergences from the anime series in terms of plot, include not only the characters, but also their traumatic battle with the Angels. Furthermore, in some instances, this strong linkage between the games and the series goes beyond genre-specific concerns as well. In the Rhythm game, 3nd Impact, on top of the cutscenes being a narrative reward, at times the music is exchanged for the characters' dialogue lines from the anime series. The sense of rhythm is thus severed from music, and control skills are employed to open up the same story once again.

The EVA games are most rewarding to fans of the anime series, as they allow for detailed comparisons. But more interestingly, as they take up the same elements obsessively and tend to reimagine a selection of the same happenings with the same characters, each of these games seems like an installment of a never-ending rewriting process. As a result, despite the fact that games are alternative producing systems in themselves, each new game seems to be an alternative to the previously released games as well as those that will probably follow. Instead of going exclusively in a medium-specific direction, the cumulation of the EVA games seems to gain a gamelike quality, supporting a specific consumer engagement.

Games certainly do not need to offer narrative experiences. Even when they feature characters whose stories are told across different media, they can centralize gamers' control skills and minimize narrative information. Their priority falls on incorporating the gamers' will and simulating the consequences of the actions they choose to 
perform. However, if combinatory play is defined as the free combination of narrative elements allowed by a suspension of causal constraints, it can be said that the EVA games as a series display a gamelikeness by featuring the same characters obsessively in combination with the same traumatic events from the series and by assigning new consequences to the same actions over a multitude of games. In this way, not only do they form a bridge with the anime series as they leave the control of the characters' actions to the gamers, but also lead the audience familiar with the anime series or the games to ask certain questions. The concern of the gamers goes beyond what they can perform in the EVA universe by stepping in the shoes of these characters, which may be seen as the medium-specific charm of games. The question instead becomes, 'How else will I be relating these characters through my actions?' This transformation reveals a narrative overtone in the player's engagement with the games and turns the experience into a form of story production (which is enabled by the games, but supervised by the consumers themselves). Individual gaming sessions thus get connected to the totality of the games and the anime series regardless of genre and (non-)narrative orientation.

This chapter aimed to expose how the EVA games are aligned with the anime series in terms of gamelikeness, and looked at the audience engagement with both media from the perspective of this alignment. In Azuma's writings, gamelikeness emerges as a point of similarity between games and narrative media, providing new ground for discussing the latter in a new light. This chapter dissected the concept of gamelikeness to underline that the similarity observed between narratives across contemporary media (i.e., the common drive toward the exploration of alternatives in narrative production and consumption) is founded on a downplay of causality, which sets narrative media once again in contrast to games. Yet again, as this discussion of the EVA games revealed, suspending medium-specific affordances is also 
an option, especially to set a particular tone in a media franchise. The EVA games seem to support a certain manner of audience engagement that is established by the anime series in the first place. The obsessive rewriting allowed by the extreme downplay of causality in the anime series finds its reflection in the serialization of the games, as an outward explosion of alternatives over a multitude of alternative generating systems. All in all, this discussion underlined not only the need to consider the medium-specificity of anime and games in relation to each other, but also the centrality of the anime series within the EVA franchise. What remains to be discussed, through comprehensive comparisons between similar franchises from all around the world, is to what extent the EVA franchise should be considered exceptional and to what extent representational of a contemporary media tendency.

\section{References}

\section{Primary sources}

3rd Impact: Grasshopper Manufacture. 20I I. Evangelion Shin GekijōbanSaundo Inpakuto. PlayStation Portable. Japanese version.

Ayanami with Asuka Raising Project: Broccoli and Gainax. 2002. Shinseiki Evangelion: Ayanami Ikusei Keikaku with Asuka Hokan Keikaku. PlayStation 2. Japanese version.

Detective Evangelion: Broccoli. 2007. Meitantei Evangelion. PlayStation 2. Japanese version.

NGE2: Bandai. 2004. Shinseiki Evangelion 2. PlayStation 2. Japanese version.

NGE 2 for PSP: Bandai. 2006. Shinseiki Evangelion 2: Tsukurareshi Sekai-Another Cases. PlayStation Portable. Japanese version.

\section{Secondary sources}

Aarseth, E. (I997). Cybertext: Perspectives on Ergodic Literature. Baltimore, MD and London: The Johns Hopkins University Press. 
Azuma, H. (2009). Otaku: Japan's Database Animals. Minneapolis, Minnesota: University of Minnesota Press. (Introduction and translation by J. E. Abel and S. Kono)

Computer Entertainment Rating Organization (CERO) website. Retrieved from: http://www.cero.biz

Costikyan, G. (20I3). Uncertainty in Games. Cambridge, Massachusetts: MIT Press.

Dengeki Online (2003). Evangerion 2 kansei kinen! Anno kantoku to Shibamura shi ga akasu Eva 2 no sekai ( 30 October). Retrieved from: https://dengekionline.com/data/news/2003/Io/30/dao6e64083 I $85 \mathrm{f} 76$ dida $5 f_{54}$ I $_{7}$ d7a8 II.html

Frasca, G. (2003). Simulation versus Narrative: Introduction to Ludology. In M. J. P. Wolf and B. Perron (eds.), The Video Game Theory Reader (pp. 23 I-235). New York and London: Routledge.

GrunGast82. (2004). Shinseiki Evangelion: Ayanami Ikusei Keikaku with Asuka Hokan Keikaku-Condition/Event Guide. Retrieved from: https://gamefaqs.gamespot.com/ps2/917346-shinseiki-evangelion -ayanami-ikusei-keikaku-with-asuka-hokan/faqs/32256

Pler, L. (2008). Neon Genesis Evangelion: Tsukurareshi Sekai-Another Cases_FAQ/Walkthrough. Retrieved from: https://gamefaqs.gamespot .com/psp/930008-neon-genesis-evangelion-tsukurareshi-sekai-another -cases/faqs/ 45735

Rancière, J. (2007). The Future of the Image. London: Verso. (Translated by G. Elliott)

Roth, M. (20I3). Playing "Naruto": Between Metanarrative Characters, Unit Operations, and Objects. In J. Berndt and B. KümmerlingMeibauer (eds.), Manga's Cultural Crossroads (pp. 243-258). New York: Routledge.

Ryan, M.-L. (2003). On Defining Narrative Media. Image [\&] Narrative, 6. Retrieved from: http://www.imageandnarrative.be/inarchive/medium theory/marielaureryan.htm

Salen, K. \& Zimmerman, E. (2004). Rules of Play: Game Design Fundamentals. Cambridge, Massachusetts: The MIT Press. 
Simons, J. (2007). Narrative, Games, and Theory. Game Studies: The International Journal of Computer Game Research, 7(I). Retrieved from: http:/gamestudies.org/07010701/articles/simons

Upton, B. (201 5). The Aesthetics of Play. Cambridge, Massachusetts: The MIT Press. 